\title{
A New Technique for Inferring Surface Albedo from Satellite Observations
}

\author{
B. PINTY \\ Université de Clermont II, L.A.M.P., 63170 Aubière, France \\ G. SZEJWACH \\ L.M.D., Ecole Polytechnique, 91128 Palaiseau, France
}

(Manuscript received 30 October 1984, in final form, March 1985)

\begin{abstract}
A technique for inferring the spatial and seasonal albedo changes over a whole climatic region from satellite data is developed. This technique uses the diurnal variation of radiances which is measured by geostationary satellites and requires the knowledge of a surface albedo value over at least one reference site. The proposed method is tested over western Africa, using METEOSAT data; and surface albedo maps representative of the wet and dry seasons are derived. With regard to the considered scales and to the achievable accuracies, the technique is shown to be relevant for climatological studies.
\end{abstract}

\section{Introduction}

In a recent paper, Henderson-Sellers and Wilson (1983) have stressed the urgent need to establish surface albedo data sets for climate modeling purposes. With regard to the sensitivity of climate models to surface albedo changes (Charney et al., 1977, Hummel and Reck, 1979), these authors have indicated that an accuracy of \pm 0.05 for albedo values was desirable at typical grid size of climate models.

Satellites appear to be the best existing tools for deriving surface albedo maps at the climatic scale, since the satellite radiance is directly related to the surface reflectance value (Slater, 1980). However, numerous effects difficult to assess (e.g., anisotropy of the reflected field at the top of the atmosphere, radiation transfer through the scattering and absorbing atmosphere, selective spectral response of the sensor) act to disrupt a simple and direct satellite retrieval of surface albedo. A detailed review of the main diffculties encountered in the use of satellites for earth radiation budget studies is given by Kandel (1983). While improvement is possible in view of correcting or avoiding some effects (Stephens et al., 1981), the problems due to atmospheric contamination are inevitable.

The removal of atmospheric contamination can be performed by theoretical calculations from the radiative transfer theory (Otterman and Fraser, 1976; Otterman, 1977). Obviously, such approach requires concurrent observations of atmospheric state, whatever scale is considered in the retrieval of surface albedo.
At present three different methods using an independent data set, together with satellite data, have been used. These methods involve specifically designed measurements of surface reflectance (Mekler and Joseph, 1983) or routine surface global radiation measurements (Pinty et al., 1985) or aircraft observations (Rockwood and Cox, 1978).

With these methods, surface albedo can be successfully derived from satellites at a mesoscale (150$200 \mathrm{~km}$ ), that is, within the one characteristic spatial scale of atmospheric transmittance. However, the retrieval of albedo maps with the same accuracy, over important climatic regions where satellite radiances are the only available data, has not been presently solved. For instance, the use of the technique proposed by Rockwood and Cox (1978) is limited to particular cases for which the atmospheric conditions remain the same as the ones prevailing during the GATE experiment. The same kind of drastic limitation exists in the alternative theoretical approach developed by Preuss and Geleyn (1980).

The goal of the technique developed here is to retrieve surface albedo from satellite radiance over a whole climatic region. This technique does not require detailed knowledge of the atmospheric state and allows a reasonable spatial variation of the atmospheric transmittance. The proposed method is used to derive albedo over western Africa and to analyze the spatial and seasonal changes of this surface parameter. The expected attainable accuracy in the derived albedo is discussed with regard to the accuracy recommended by climate modelers. 


\section{Albedo estimate technique}

The proposed technique is based on the estimate of the reflectance ratios between several pairs of neighboring areas; these reflectance ratios are deduced from the diurnal cycles of radiances measured by a geostationary satellite under clear-sky situations only.

For an area ( $i)$, observed under cloudless conditions, the effective spectral radiance is given by the following equation (Slater, 1980):

$$
\begin{array}{r}
L_{\lambda s, i}\left(\theta_{0}, \theta_{v}, \phi\right)=F(\lambda)\left[\frac{1}{\pi} E_{\lambda, i}\left(\theta_{0}\right) \rho_{i}\left(\lambda, \theta_{0}, \theta_{v}, \phi\right)\right. \\
\left.\times \tau_{i}\left(\lambda, \theta_{v}\right)+L_{\lambda u, i}\left(\theta_{0}, \theta_{v}, \phi\right)\right]
\end{array}
$$

where $\theta_{0}, \theta_{v}$ and $\phi$ denote the solar zenith angle, the satellite viewing angle and the relative azimuth angle, respectively. In Eq. (1), $L_{\lambda s, i}\left(\theta_{0}, \theta_{v}, \phi\right)$ is the spectral radiance measured by the sensor, $E_{\lambda, i}\left(\theta_{0}\right)$ is the surface spectral irradiance, $\tau_{i}\left(\lambda, \theta_{v}\right)$ the spectral atmospheric transmittance, $\rho_{i}\left(\lambda, \theta_{0}, \theta_{v}, \phi\right)$ the surface spectral reflectance, $L_{\lambda u, i}\left(\theta_{0}, \theta_{v}, \phi\right)$ the spectral atmospheric path radiance and $F(\lambda)$ sensor spectral response.

For two neighboring areas $(i)$ and $(j)$ observed during a whole day under similar atmospheric conditions, we can write:

$$
\begin{gathered}
\tau_{j}\left(\lambda, \theta_{v}\right)=\tau_{i}\left(\lambda, \theta_{v}\right)+\epsilon_{\tau_{i, j}}\left(\lambda, \theta_{v}\right) \\
E_{\lambda, j}\left(\theta_{0}\right)=E_{\lambda, i}\left(\theta_{0}\right)+\epsilon_{E_{\lambda, i j}}\left(\theta_{0}\right) .
\end{gathered}
$$

Therefore, by omitting the angular dependence, $L_{\lambda s, j}$ and $L_{\lambda s, i}$ are related by Eq. (4):

$$
\begin{aligned}
L_{\lambda s, j}= & \frac{\rho_{j}(\lambda)}{\rho_{i}(\lambda)}\left(1+\frac{\epsilon_{\tau_{i, j}}(\lambda)}{\tau_{i}(\lambda)}\right) L_{\lambda s, i}+L_{\lambda u, j} F(\lambda) \\
& -\frac{\rho_{j}(\lambda)}{\rho_{i}(\lambda)}\left(1+\frac{\epsilon_{i, j}(\lambda)}{\tau_{i}(\lambda)}\right) L_{\lambda u, i} F(\lambda) \\
& +\frac{1}{\pi} \rho_{j}(\lambda) \tau_{j}(\lambda) \epsilon_{E_{\lambda, i, j}} F(\lambda) .
\end{aligned}
$$

By integrating Eq. (4) over $\lambda$ between $\lambda_{1}$ and $\lambda_{2}$, (the lower and upper wavelength limits of the sensor response) and using the integral mean value theorem, we can derive Eq. (5):

$$
\begin{array}{r}
L_{s, j}=\frac{\rho_{j}\left(\lambda^{*}\right)}{\rho_{i}\left(\lambda^{*}\right)}\left[1+\frac{\epsilon_{\tau_{i, j}}\left(\lambda^{*}\right)}{\tau_{i}\left(\lambda^{*}\right)}\right] L_{s, i}+\int_{\lambda_{1}}^{\lambda_{2}} L_{\lambda u, j} F(\lambda) d \lambda \\
-\frac{\rho_{j}\left(\lambda^{*}\right)}{\rho_{i}\left(\lambda^{*}\right)}\left[1+\frac{\epsilon_{\tau_{i, j}}\left(\lambda^{*}\right)}{\tau_{i}\left(\lambda^{*}\right)}\right] \int_{\lambda_{1}}^{\lambda_{2}} L_{\lambda u, i} F(\lambda) d \lambda \\
+\frac{1}{\pi} \int_{\lambda_{1}}^{\lambda_{2}} \rho_{j}(\lambda) \tau_{j}(\lambda) \epsilon_{E_{\lambda, i}, j} F(\lambda) d \lambda
\end{array}
$$

with $\lambda_{1}<\lambda^{*}<\lambda_{2}$.
As long as, the characteristic spatial scale of atmospheric transmittance (typically 100 to $200 \mathrm{~km}$ under clear sky conditions) remains larger than the basic size of the considered areas, $\epsilon_{\tau_{i, j}}$ and $\epsilon_{E_{\lambda, i}, j}$ are close to zero. With the physically reasonable assumption that the spatial difference of sky effects (second + third terms) is constant during a clear sky day, then the reflectance ratio $\rho_{j}\left(\lambda^{*}\right) / \rho_{i}\left(\lambda^{*}\right)$ can be derived from a linear regression between the respective radiances $L_{s, j}$ and $L_{s, i}$ measured during a diurnal cycle by a geostationary satellite.

Similarly reflectance ratios $\rho_{j} / \rho_{k}, \rho_{k} / \rho_{l}, \ldots$ can be obtained by considering other pairs of adjacent areas until coverage of the considered climatic region is completed. The absolute reflectance values of each area can then be obtained from the knowledge of one only of the reflectances considered in the series of reflectance ratios.

Since very little data is available concerning the bidirectional properties of the region investigated in this study, we will assume that the surface is a Lambertian reflector and from now on we will consider that the derived reflectance values are representative of the surface albedos.

According to Eq. (5), the presence of a spatial gradient in transmittance alters the estimate of surface albedos by the proposed ratio technique. The error in albedo estimate of the $n$th area which arises from application of the ratio technique considering an hypothetical constant horizontal gradient in transmittance can be approximated as follows:

$$
\hat{\rho}_{n}=\rho_{n}(1+n \epsilon / \tau)
$$

where $\hat{\rho}_{n}$ and $\rho_{n}$ are the estimated and the true surface albedo of the $n$th area, respectively; $\epsilon / \tau$ is the relative spatial variation in atmospheric transmittance.

The relative error $(\Delta \rho / \rho)$ in albedo given by Eq. (6) is illustrated in Fig. 1 for different values of the relative spatial variation in transmittance $(\epsilon / \tau)$. Figure la shows that if a $25 \%$ relative accuracy in the retrieved surface albedo is desirable over an area located $1000 \mathrm{~km}$ away from the reference site, then the mean relative spatial variation in transmittance must not exceed $3 \% / 100 \mathrm{~km}$ over the considered region. In the case of a relative spatial variation in transmittance of $5 \% / 100 \mathrm{~km}$, the relative uncertainty of $25 \%$ in surface albedo will be reached at a distance of $500 \mathrm{~km}$ from the reference site location. Figure $1 \mathrm{~b}$ illustrates the atmospheric conditions leading to 0.05 and 0.1 absolute accuracies in albedo estimate when the ratio technique is applied over a region with an hypothetical spatial gradient and reference albedo values of 0.04 for each basic area and 0.2 , respectively. The dashed curves correspond to the case of an albedo gradient equals to -0.04 with a reference value of 0.6. It appears that for a given set of absolute albedo accuracies, the atmospheric conditions allowing 


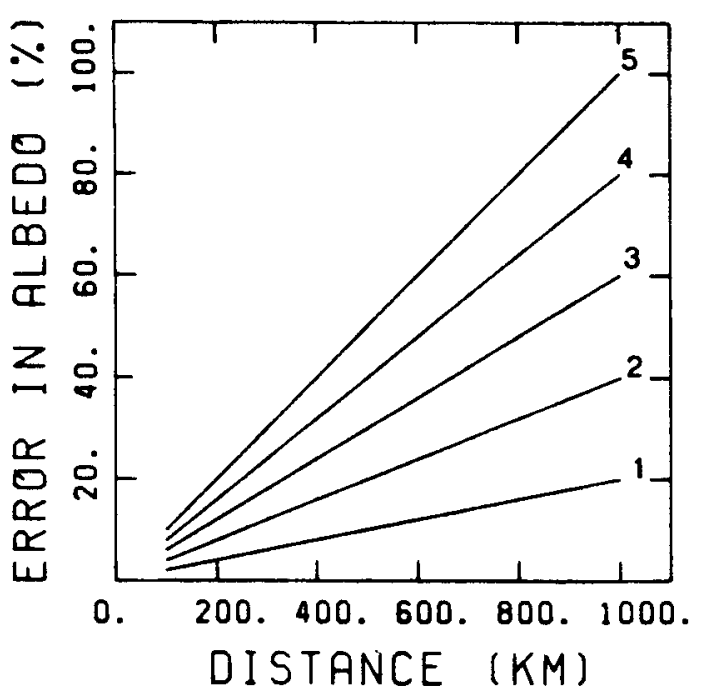

FIG. 1a. Relative error in retrieved surface albedo vs. the distance from the reference site, for the five following values of relative spatial variation in transmittance: 1) $\left.2 \%(100 \mathrm{~km})^{-1}, 2\right) 4 \%(100$ $\mathrm{km})^{-1}$, 3) $6 \%(100 \mathrm{~km})^{-1}$, 4) $8 \%(100 \mathrm{~km})^{-1}$, 5) $10 \%(100 \mathrm{~km})^{-1}$.

the use of the ratio technique are different from the value of the albedo reference-that is, the relative geographical location of the reference site. In other words, for a given climatic region, the reference site must be chosen in order to minimize the error arising from an expected (from ground data for instance) transmittance gradient at a large scale.

\section{Data selection and processing}

As mentioned in Section 2, the ratio technique applies only to clear sky situations. In practice, it is important to note that the occurrence of such conditions over whole climatic regions is rather sparse and it remains difficult to ascertain that a truly clear sky situation exists from satellite data alone. Nevertheless, infrared satellite pictures allow a rough identification of cloudy scenes at scales larger than the pixel size, and in situ measurements of visibility from the meteorological network can be used to examine the occurrence of extended dust clouds.

In order to examine the spatial and seasonal albedo changes over West Africa, METEOSAT-1 visible data for 18 February and 2 July 1979 have been retained. We will assume that these days are representative of typical surface conditions for the wet and dry seasons, respectively. For these two days, a large part of West Africa appears cloud free on visible and infrared pictures. For 2 July, a little doubt exists about the aerosol concentration over western Mauritania, since the climatological bulletin from ASECNA Office mentioned the occurrence of Saharian dust at the beginning of the month over this region. Nevertheless, we have tried to apply the ratio technique for the 2 July case, assuming that the hypothesis related to atmospheric transmission was satisfied.

In the present study, the conversion of METEOSAT radiances to broadband radiances has not been performed since little is known about the METEOSAT1 conversion factor. This factor is related to observational geometry, spectral variation in optical depth, surface reflectance, but the value to be considered for each pixel of a given radiance map remains unknown. Koepke (1983) indicates that in the case of METEOSAT-2, the conversion factor is $2.8 \pm 1$ for vegetated surfaces and $2.4 \pm 1$ for bare surfaces and dry vegetation. From the results of that paper, one can assume that the conversion factor decreases with increasing albedo. As the difference between the spectral response of the visible channels of METEOSAT-1 and METEOSAT-2 is less than 1\% (Koepke, 1982), the above assumption can be made as well for METEOSAT-1 data. In our application, the conversion factor is kept constant over the whole West Africa region; therefore we can expect that our METEOSAT albedos overestimate the total solar spectrum-weighted albedo over regions of relatively high albedo values.

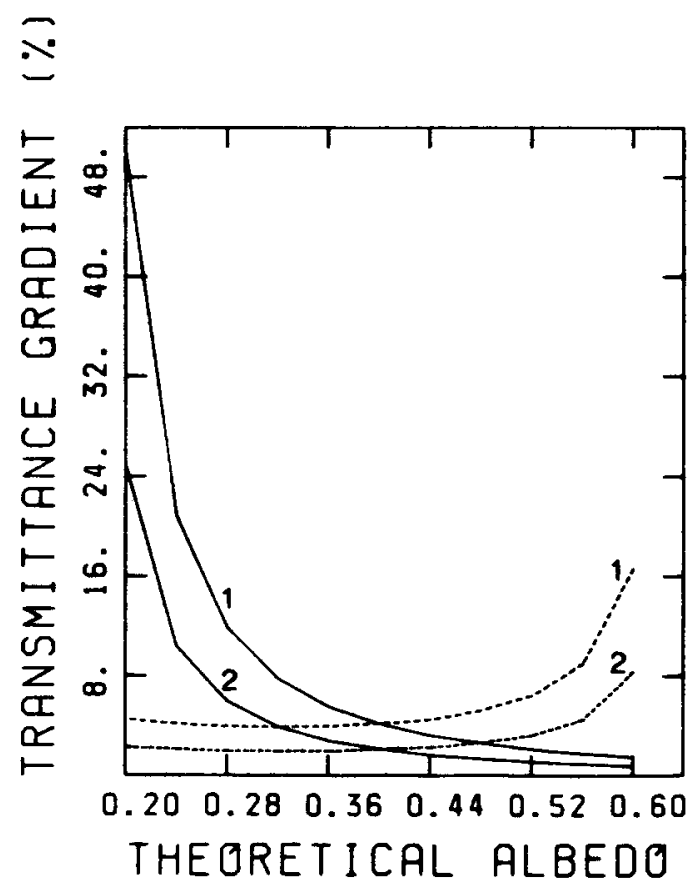

FIG. 1b. Relative spatial variation in transmittance limiting the absolute accuracy of the derived albedos to 0.10 (curves 1 ) and 0.05 (curves 2), respectively. Full curves correspond to the case of a reference value $\rho=0.2$ and an albedo gradient $\partial \rho / \partial n=0.04$ per basic area; dashed curves are for $\rho=0.6$ and $\partial \rho / \partial n=-0.04$ per basic area. 


\section{Technique validation}

The proposed ratio technique has been tested over Upper-Volta with METEOSAT data for 18 February and 2 July 1979. We first independently derived the $50 \mathrm{~km} \times 50 \mathrm{~km}$ surface albedo around three test sites: Dori $\left(14.05^{\circ} \mathrm{N}, 0^{\circ}\right)$, Ouagadougou $\left(12.42^{\circ} \mathrm{N}\right.$, $\left.1.5^{\circ} \mathrm{W}\right)$ and Fada-Ngourma $\left(12.06^{\circ} \mathrm{N}, 0.4^{\circ} \mathrm{E}\right)$ following the method described by Pinty et al. (1985). This method uses simultaneous measurements of satellite radiances and routine surface global radiation. It allows an estimate of surface albedo at a mesoscale over each site where the required measurements are available. According to the results presented in that previous study, a relative uncertainty of about 10 to $20 \%$ can be reasonably expected for these albedo estimates. These values (Table 1) will be now referred to as reference albedos.

To test the proposed method, we computed the surface albedos of the three test sites using the ratio technique and compared the results to the reference value. The three reference values are used successively to initiate the process. The results presented in Table 2 indicate that the ratio technique gives a consistent albedo estimate for each location. On 18 February values obtained starting from Ouagadougou are somewhat larger than the reference value independently estimated at this site; however, in the worst case the difference does not exceed $15 \%$ and remains within the uncertainty involved in the estimate of the reference value. Despite the different values in the transmittances observed at the three test sites (Table 1), no significant increase in the quality of these estimates can be detected when using different paths in the ratio technique.

Figures $2 \mathrm{a}$ and $2 \mathrm{~b}$ represent albedo maps of the northern Upper-Volta for 18 February and 2 July, respectively, obtained using the ratio technique with Fada-Ngourma as a reference site. The vertical shading and numbers on the right-hand side of the figure represent the scale. The black squares correspond to regions where the technique is not applicable, due to local cloud contamination, for instance. It can be noted that results shown in Table 2 are consistent, despite the large spatial variability of derived albedos between the three test sites.

TABLE 1. Transmittance and albedo estimates at the test sites.

\begin{tabular}{lccccc}
\hline \hline & \multicolumn{2}{c}{ February } & & \multicolumn{2}{c}{ July } \\
\cline { 2 - 3 } \cline { 5 - 6 } \cline { 5 - 6 } Test sites & $\begin{array}{c}\text { Observed } \\
\text { trans- } \\
\text { mittance }\end{array}$ & $\begin{array}{c}\text { Estimated } \\
\text { albedo }\end{array}$ & $\begin{array}{c}\text { Observed } \\
\text { trans- } \\
\text { mittance }\end{array}$ & $\begin{array}{c}\text { Estimated } \\
\text { albedo }\end{array}$ \\
\hline (1) Dori & 0.747 & 0.379 & & 0.623 & 0.375 \\
(2) Ouagadougou & 0.695 & 0.308 & & 0.650 & 0.285 \\
(3) Fada-Ngourma & 0.703 & 0.224 & 0.665 & 0.279 \\
\hline
\end{tabular}

TABLE 2. Test-results of the ratio technique. In each line, the site values taken as reference are underlined. The first line shows the ratio technique results obtained at Ouagadougou site and FadaNgourma site when using Dori site values to initiate the process. Results in the two following lines are obtained when using Ouagadougou site values and Fada-Ngourma site values to initiate the ratio technique process, respectively.

\begin{tabular}{cccccccc}
\hline \hline \multicolumn{2}{c}{ Dori } & & \multicolumn{2}{c}{ Ouagadougou } & & \multicolumn{2}{c}{ Fada-Ngourma } \\
February & July & & February & July & & February & July \\
\hline$\underline{0.379}$ & $\underline{0.375}$ & & 0.271 & 0.290 & & 0.220 & 0.276 \\
0.431 & 0.368 & & $\underline{0.308}$ & $\underline{0.285}$ & & 0.250 & 0.271 \\
0.387 & 0.379 & & $\mathbf{0 . 2 7 6}$ & $\underline{0.293}$ & & $\underline{0.224}$ & $\underline{0.279}$ \\
\hline
\end{tabular}

\section{Results}

\section{a. Surface albedo maps}

Surface albedo maps were derived over northwestern Africa using the ratio technique (Figs. 3a and 3b). Each square represents an average albedo value over $20 \times 20$ pixels (approximately $100 \mathrm{~km} \times 100$ $\mathrm{km})$. The reference albedos have been estimated at the corresponding scale at Fada-Ngourma for 18 February and 2 July. In the studied cases, scarcity of ground data which are essential to derive a satellite reference albedo has limited the estimate of reference albedos to Upper-Volta sites. The albedo maps were obtained from sets of 10 pictures and 7 pictures between 1030 and 1500 GMT for 18 February and 2 July, respectively. It must be recalled that the term "albedos" refers to the reflectance integrated over the METEOSAT sensor spectral response (0.4-1.1 $\mu \mathrm{m})$.

Retrieved albedo values range from 0.18 to 0.6 . High values (greater than 0.4 ) which are located over the central and northeastern part of the maps correspond to the desertic regions of Mali and Mauritania. Values reaching 0.6 are observed at the northeastern corner of the maps, over the Algerian ergs and the Tademait Plateau. Slightly to the south of this region, an isolated zone of relatively lower albedos corresponds to the location of the oasis of Tidikelt. A pronounced north-south and east-west gradient in albedo over West Africa is observed. With the onset of the wet season, a general decrease in albedo is observed west of $5^{\circ} \mathrm{W}$. During the same time, south of $20^{\circ} \mathrm{N}$, the $40 \%$ line of albedo migrates from 10 to $5^{\circ} \mathrm{W}$, while at the north of $20^{\circ} \mathrm{N}$, this line does not exhibit any displacement.

The general patterns of our albedo maps agree reasonably well with those derived by Rockwood and Cox (1978), Norton et al. (1979) and Courel et al. (1984), keeping in mind that data sets, methods and sensor responses are different for all of these studies. The major difference occurs over desertic regions where our values are somewhat higher than the ones previously derived. As discussed in Section 3, high 
METEOSAT albedo regions can be biased by the particular shape and range of the METEOSAT visible channel. If we consider that the values of 2.8 and 2.4 given by Koepke (1983) for the METEOSAT conversion factor can be applied over the reference site and highest albedo regions, respectively, then these high albedo values will be reduced by approximately $15 \%$. As pointed out by Courel et al., (1984) and in spite of the broadband correction, the derived albedos over the Sahara are significantly higher than the values usually considered for climate modeling purposes.

\section{b. Seasonal albedo changes}

Figures $4 \mathrm{a}$ and $4 \mathrm{~b}$ represent the positive and negative albedo changes expressed in percent between 18 February and 2 July 1979.

$$
S V(\%)=\left(\rho_{\mathrm{Jul}}-\rho_{\mathrm{Feb}}\right) / \rho_{\mathrm{Feb}} \times 100 .
$$

Positive values of $S V$ reaching $15 \%$ are located over the Sahara desert. Since no appreciable seasonal change can be expected in this region, this relative albedo increase is certainly related to uncertainties associated with our technique. Over the same region, Courel et al. (1984) found a variation of $15 \%$ between 1973 and 1979.

The well-known general decrease in albedo over the Sahel between the dry and wet seasons is clearly depicted in Fig. 4b. The maximum albedo decreases reaching $40 \%$ are found between the Atlantic Ocean coast and $10^{\circ} \mathrm{W}$. In comparison a much weaker decrease is observed over central and eastern Sahel. Such a pattern of seasonal albedo changes is in good agreement with the results of Norton et al. (1979).

\section{Estimated accuracies of the derived albedos}

Besides the assumption that the reflected radiance field at the top of the atmosphere is isotropic, there are mainly three sources of error leading to inaccuracies in our albedo maps: 1) the use of an erroneous albedo reference value; 2) the presence of a largescale gradient in atmospheric transmittance; and 3) natural surface heterogeneities, to the extent that no significant spatial average for albedos can be obtained.

The first point has been extensively discussed in Pinty et al. (1985) and, in practice, a relative accuracy better than $20 \%$ of the retrieved value is expected. Obviously, the error existing in the reference albedo estimate propagates over the whole climatic region when the ratio technique is used.

We have discussed in Section 2 the hypothetical albedo error involved by an application of the ratio technique under a constant large-scale gradient in atmospheric transmittance. For the cases considered in this paper, neither visible nor infrared METEOSAT data, nor surface routine meteorological data seem to indicate a marked transmittance gradient over West Africa. The eventuality of an aerosol content increase over western Mauritania for the 2 July case would lead to an underestimate of surface albedo derived by the ratio technique over this region of relatively low albedo values. However, with regard to the use of the ratio technique, such errors due to particular atmospheric conditions should be cancelled by an estimate of albedo values for several days during the same season.

Each of our albedo values has been derived over a size area of $100 \mathrm{~km} \times 100 \mathrm{~km}$ approximately. Since these estimates use spatially averaged radiances, natural heterogeneities inside each area limit the representativeness of each resulting albedo value. Figures $5 \mathrm{a}$, and $5 \mathrm{~b}$ present the albedo standard deviations $\sigma_{\rho}$ computed for each $20 \times 20$ pixel area using Eq. (8) from Pinty et al. (1985):

$$
\sigma_{\rho} \approx \sigma_{L s} \pi \frac{E_{s}}{E_{G}{ }^{2}}\left(1-4 \alpha_{s}\langle\rho\rangle\right)^{-1 / 2}
$$

where $\sigma_{\rho}$ and $\sigma_{L s}$ are the albedo standard deviation and radiance standard deviation, respectively; $E_{s}$ denotes the solar irradiance at the top of the atmosphere, $E_{G}$ is the surface global radiation and $\alpha_{s}$ is the spherical albedo of the atmosphere. The brackets represent a spatially averaged value.

Equation (8) expresses in a simplified manner the contrast reduction due to the presence of the atmosphere. Values obtained from Eq. (8) for $\sigma_{\rho}$ range from 0.02 to 0.16 . It is interesting to note that Figs. $5 \mathrm{a}$, and $5 \mathrm{~b}$ show similar patterns, particularly in regions of high albedos $(\rho \geqslant 0.35)$. The fact that we observe a good correlation between both maps indicate that for the considered scale $(100 \mathrm{~km} \times 100 \mathrm{~km})$ the natural heterogeneity of the surface albedo does not vary significantly. These maps represent also a good quantitative estimate of the expected accuracy attainable for the albedo at the climatic scale with a METEOSAT sensor.

All these errors can be simultaneously present and it is difficult to estimate the resulting inaccuracy for albedo since, for instance, errors arising from the first and second effects can cancel each other out.

In practice, the error related to our surface albedos can be estimated assuming that the albedo averaged over the desertic zone $\left(19-28^{\circ} \mathrm{N}, 4^{\circ} \mathrm{E}-4^{\circ} \mathrm{W}\right)$ remains unchanged from February to July months. Over this zone, the albedo difference obtained from the two independent data sets is 0.03 . Although from a statistical point of view and in extreme cases the resulting error would be greater, this value can be taken as an indicator of the effective albedo accuracy which is attainable with the ratio technique. 


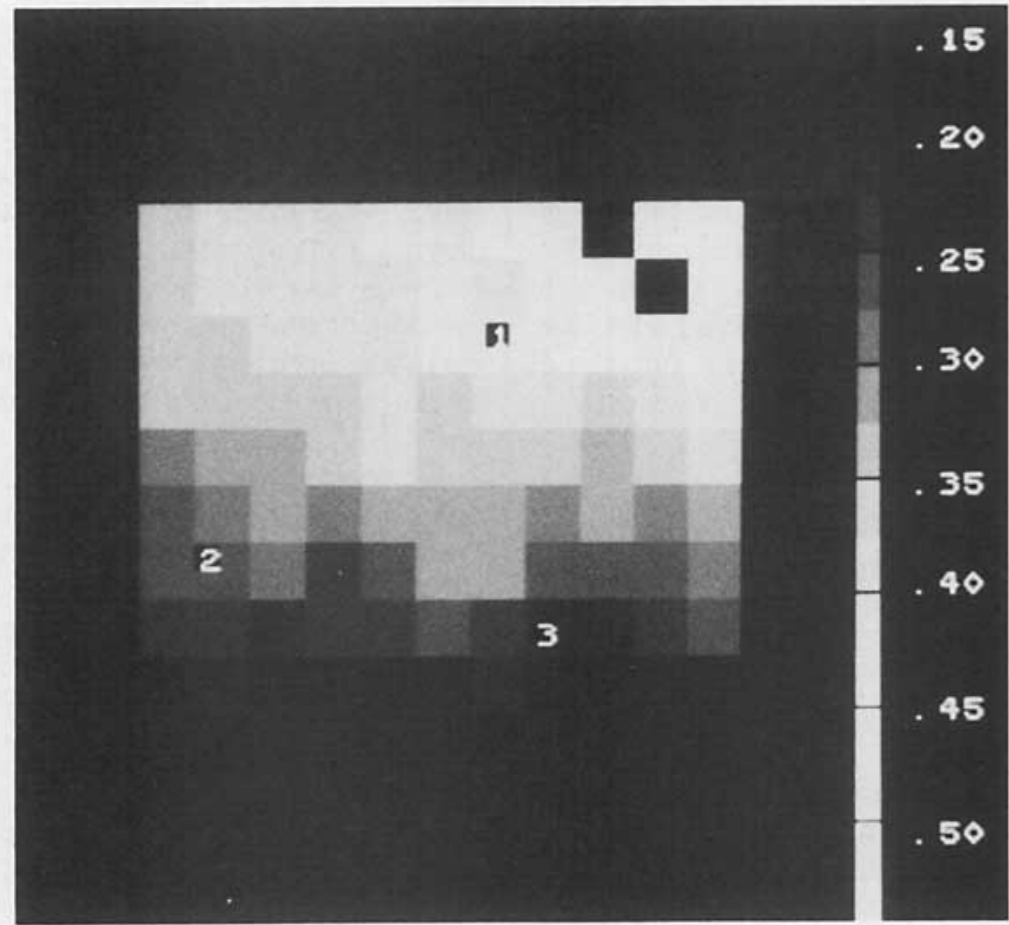

FIG. 2a. Albedo map for 18 February, 1979 of the northern Upper-Volta. Sites identification: 1-Dori; 2-Ouagadougou; 3-Fada-Ngourma. (The grey scale is indicated on the right-hand side).

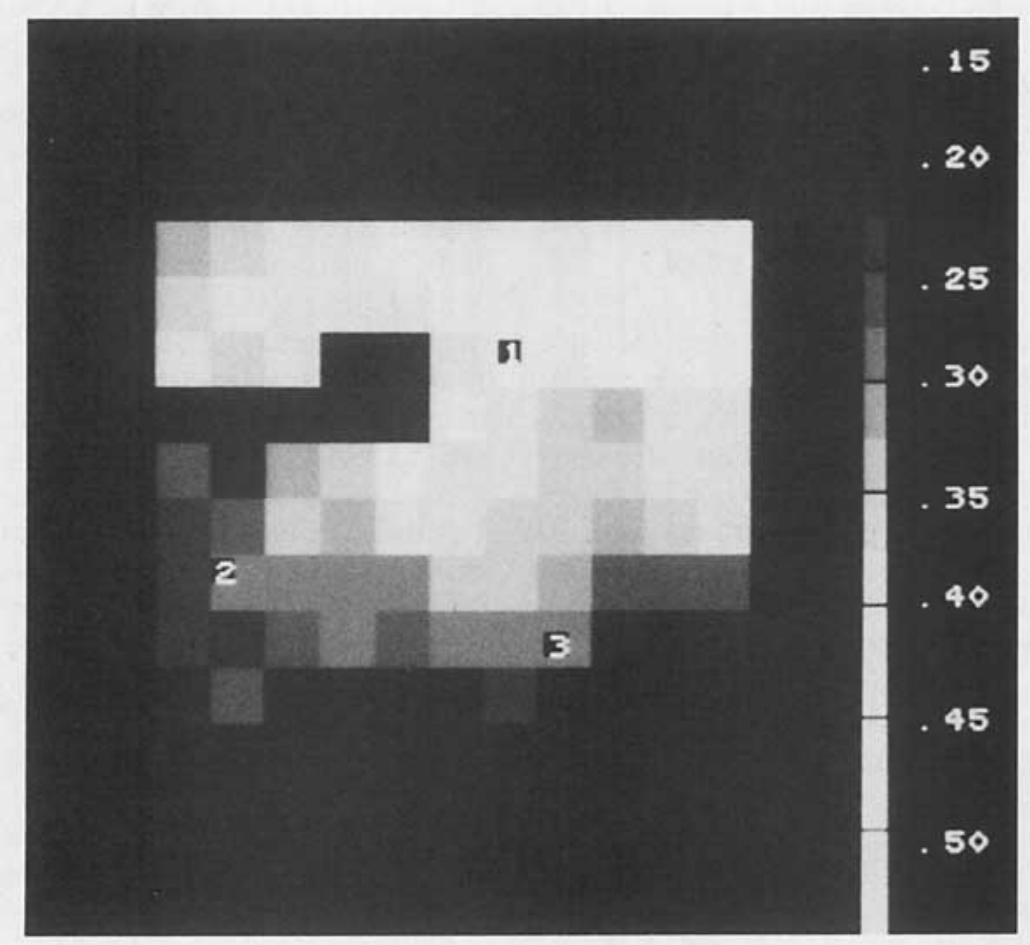

FIG. 2b. As in Fig. 2a except for 2 July 1979. 


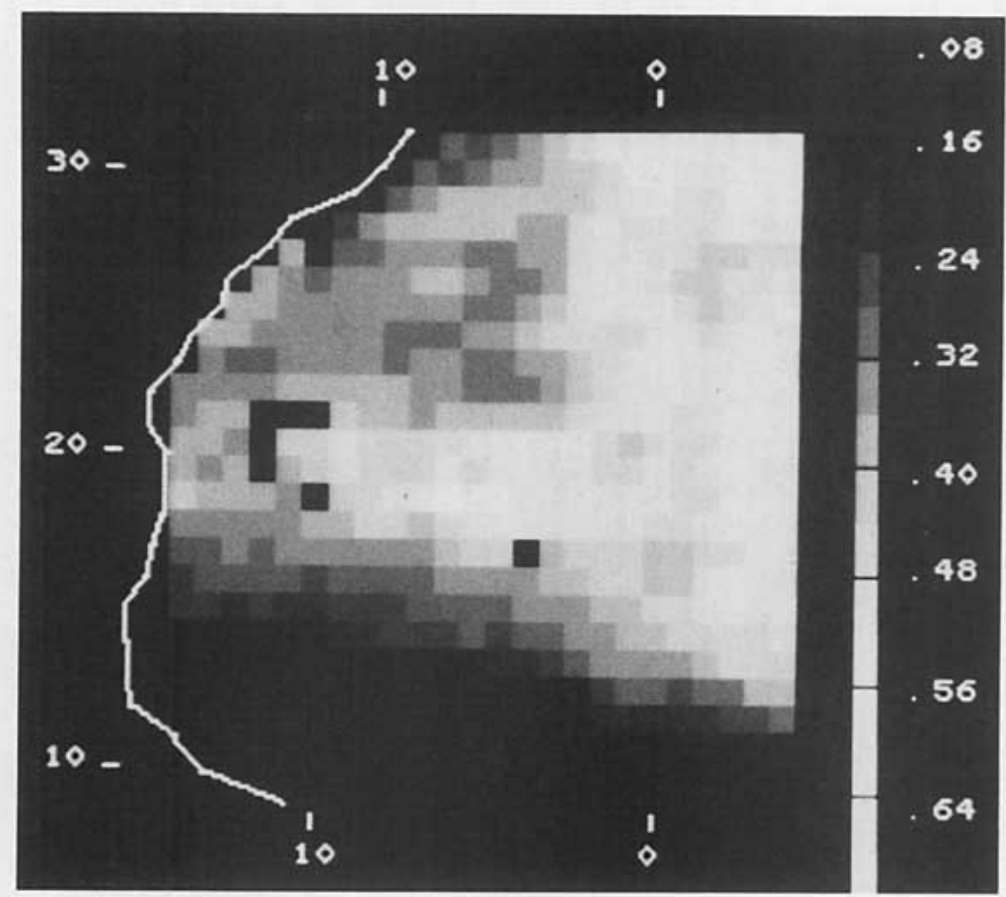

FIG. 3a. Surface albedo map for 18 February 1979.

(The grey scale is indicated on the right-hand side).

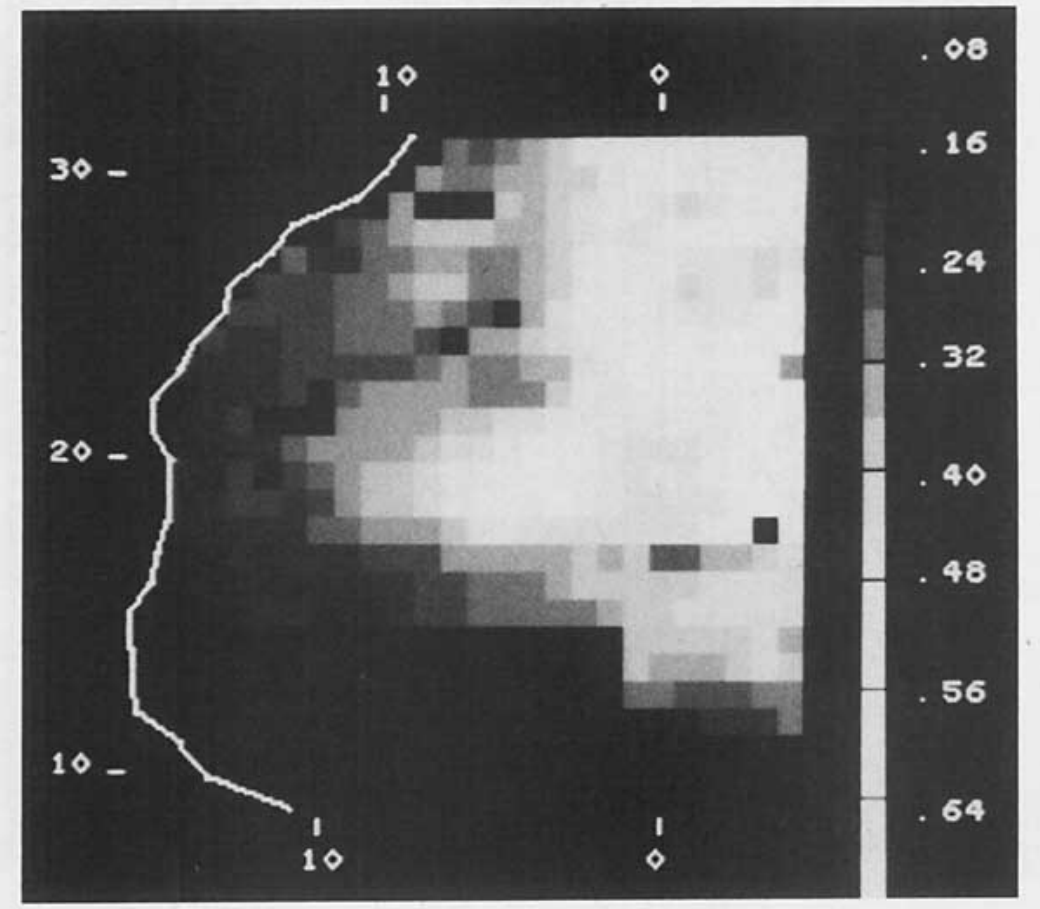

FiG. 3b. Surface albedo map for 2 July 1979 


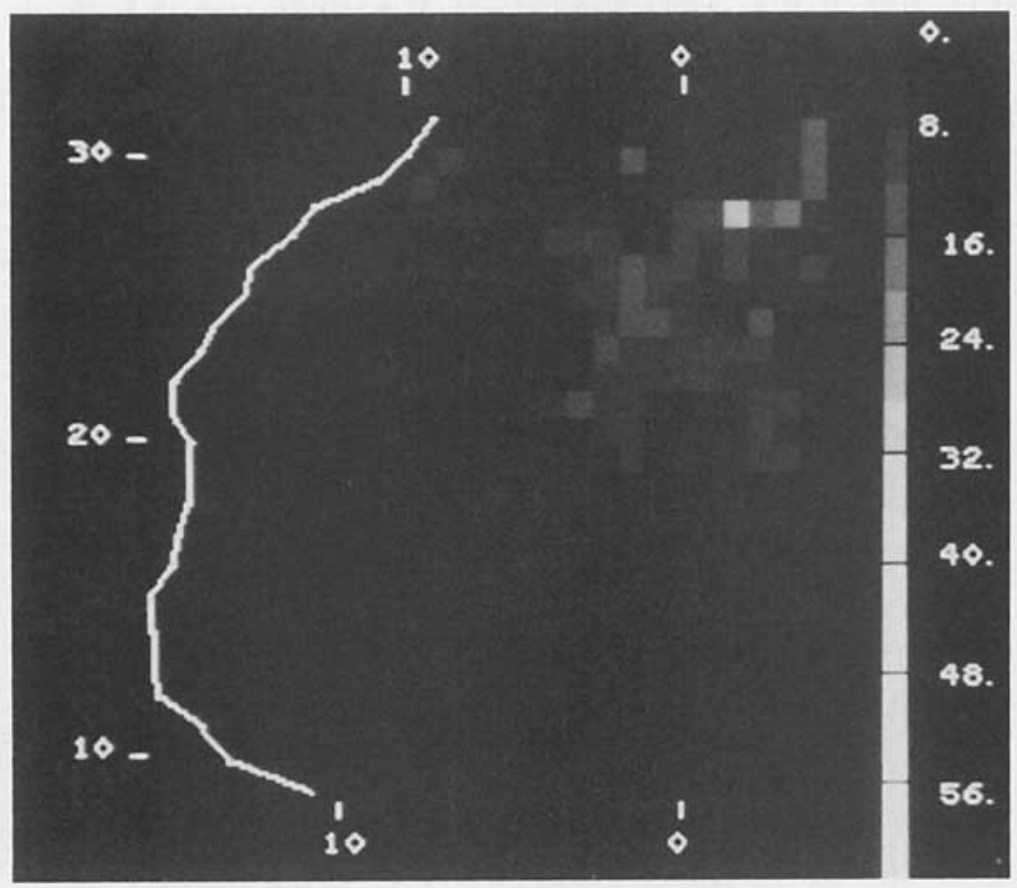

FIG. 4a. Positive change (in percent) in surface albedo between 2 July and 18 February 1979. (The grey scale is indicated on the right-hand side).

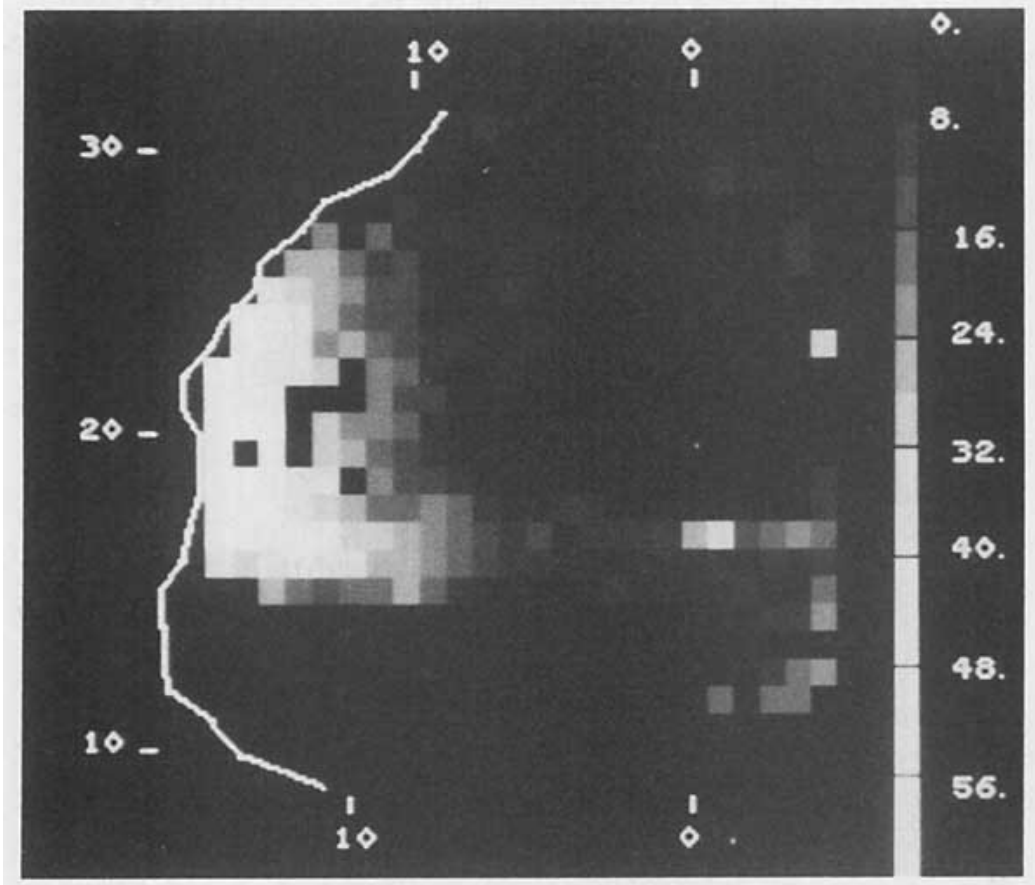

FIG. 4b. Negative change (in percent) in surface albedo between 2 July and 18 February 1979. 


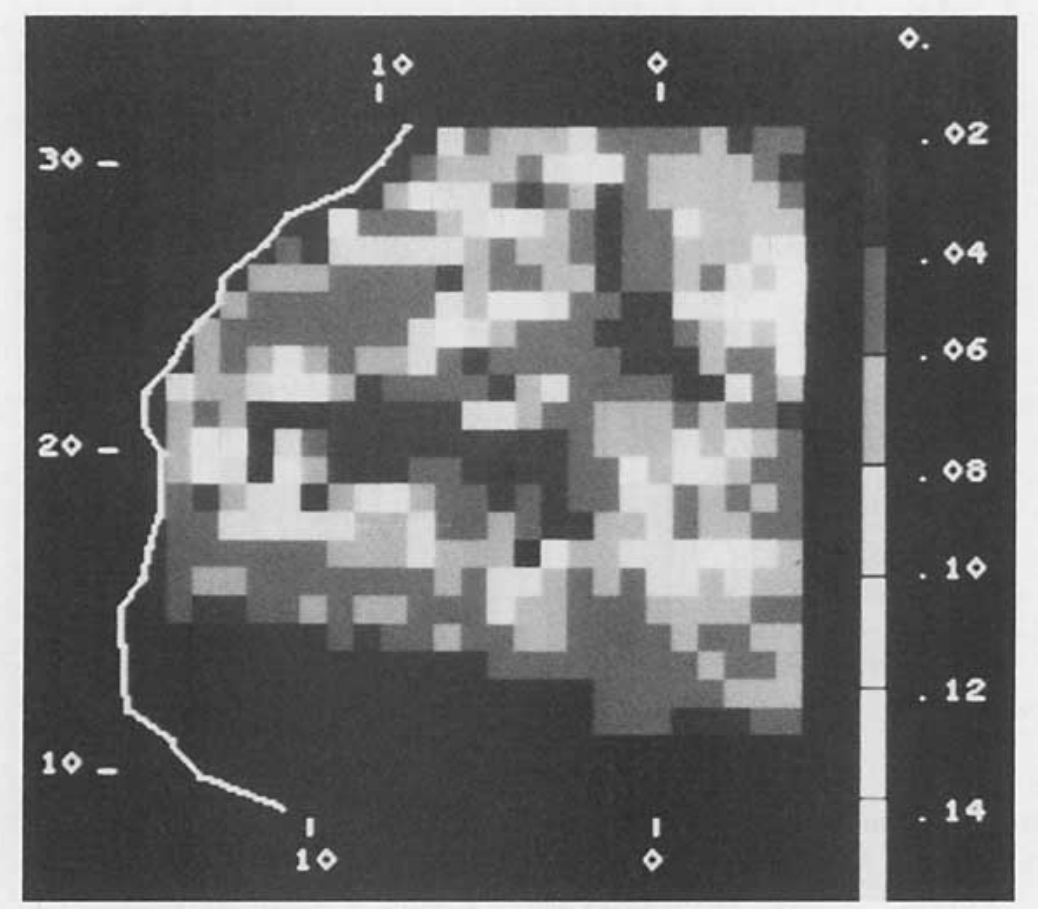

FIG. 5a. Albedo standard deviation map for 18 February 1979. (The grey scale is indicated on the right-hand side).

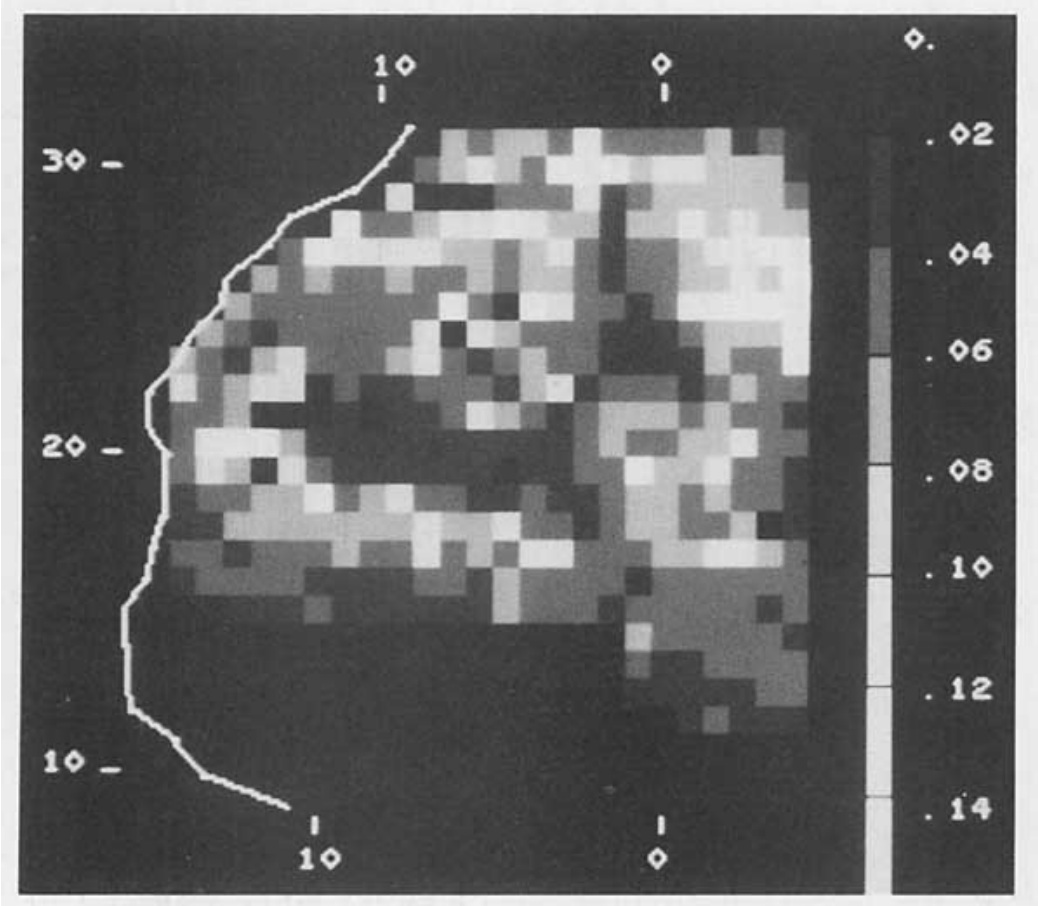

FIG. 5b. Albedo standard deviation map for 2 July 1979. 


\section{Conclusions}

A technique has been developed to infer surface albedo maps from geostationary satellite data over a whole climatic region. This technique uses several measurements over the same region during one day. Its application is limited to clear-sky conditions and requires the knowledge of at least one surface reference site.

The method has been successfully tested over Upper-Volta for three reference test sites using METEOSAT-1 measurements. Data from this geostationary satellite have allowed albedo maps to be derived over western Africa, for two days representative of the dry and wet seasons, respectively. The well-known seasonal change related to the growth of vegetation with the onset of the wet season has been quantitatively estimated. According to previous studies, an albedo decrease between February and July has been observed west of $9^{\circ} \mathrm{W}$ and south of $18^{\circ} \mathrm{N}$, The remaining problem with the METEOSAT derived albedos is that these values must be converted into total solar spectrum-weighted albedos.

With regard to accuracy of the technique, the estimated errors in the derived albedos appear acceptable for climatic modeling purposes. Furthermore, the technique allows an estimate of albedo values with a spatial resolution compatible with one used in climate models. A great advantage of the proposed technique is that detailed properties of the atmosphere need not be known over the investigated region. Since Pinty et al. (1985) have shown that albedo reference sites can be defined with the help of existing routine surface data, such approach allows a simple satellite monitoring of surface albedo.

Acknowledgments. The authors are indebted to Drs. H. Isaka, D. Ramond and M. Desbois for their encouragement and support. Special thanks are due to Dr. Henderson-Sellers and Dr. P. Waldteufel for helpful suggestions about the manuscript. We are also grateful to R. Pejoux for his computer assistance and we thank C. Paquet for typing the manuscript and $J$. Squarise for editing aid.

\section{REFERENCES}

Charney, J. G., W. J. Quirk, S. H. Chow and J. Kornfield, 1977: A comparative study of the effects of albedo change on drought in semi-arid regions. J. Atmos. Sci., 34, 1366-1385.

Courel, M. F., R. S. Kandel and S. I. Rasool, 1984: Surface albedo and the Sahel drought. Nature, 307, 528-531.

Henderson-Sellers, A., and M. F. Wilson, 1983: Surface albedo data for climatic modeling. Rev. Geophys. Space. Phys., 21, 1743-1778.

Hummel, J. R., and R. A. Reck, 1979: A global surface albedo model. J. Appl. Meteor., 18, 239-253.

Kandel, R. S., 1983: Satellite Observation of the earth radiation budget. Beitr. Phys. Atmos., 56, 322-340.

Koepke, P., 1982: Meteosat-VIS-channel: signal reduction to atmospheric water vapor and ozone. Beitr. Phys. Atmos., 55, 358-369.

- 1983: Calibration of the VIS-channel of Meteosat-2. Advances in Space Research., Vol. 2, 93-96.

Mekler, Y., and J. H. Joseph, 1983: Direct determination of surface albedos from satellite imagery. J. Climate Appl. Meteor., 22, 530-536.

Norton, C. C., F. R. Mosher and B. Hinton, 1979: An investigation of surface albedo variations during the recent Sahel drought. J. Appl. Meteor., 18, 1252-1262.

Otterman, J., 1977: Monitoring surface albedo changes with LANDSAT. Geophys. Res. Lett., 4, 441-448.

- , and R. S. Fraser, 1976: Earth atmosphere system and surface reflectivities in regions from LANDSAT MSS measurements. Remote Sens. Environ., 5, 247-266.

Pinty, B., G. Szejwach and J. Stum, 1985: Surface albedo over the Sahel from METEOSAT radiances. J. Climate Appl. Meteor., 24, 108-113.

Preuss, H., and J. F. Geleyn, 1980: Surface albedoes derived from satellite data and their impact on forecast models. Arch. Meteor. Geophys. Bioklim. Ser. B, 29, 345-356.

Rockwood, A. A., and S. K. Cox, 1978: Satellite inferred surface albedo over Northwestern Africa. J. Atmos. Sci., 35, 513-522.

Slater, P. N., 1980: Remote sensing. Optics and optical systems. Ed., Simonett, D. S., Addison-Wesley, 575 pp.

Stephens, G. L., G. G. Campbell and T. H. Vonder Haar, 1981: Earth radiation budget. J. Geophys. Res., 86, 9739-9760. 